\title{
Buprenorphine for Medication-Assisted Treatment of Opioid Use Disorder in Pregnancy: Relationship to Neonatal Opioid Withdrawal Syndrome
}

\author{
Niraj R. Chavan, MD, MPH ${ }^{1}$ Kristin B. Ashford, PhD, WHNP-BC ${ }^{2}$ Amanda T. Wiggins, PhD $^{2}$ \\ Michelle R. Lofwall, MD ${ }^{3}$ Agatha S. Critchfield, MD ${ }^{1}$ \\ ${ }^{1}$ Division of Maternal Fetal Medicine, Department of Obstetrics and \\ Gynecology, University of Kentucky College of Medicine, \\ Lexington, Kentucky \\ 2 University of Kentucky College of Nursing, Lexington, Kentucky \\ ${ }^{3}$ Department of Behavioral Science and Psychiatry, Center on Drug \\ and Alcohol Research, University of Kentucky College of Medicine, \\ Lexington, Kentucky \\ Am J Perinatol Rep 2017;7:e215-e222. \\ Address for correspondence Niraj Chavan, MD, MPH, Division of \\ Maternal Fetal Medicine, Department of Obstetrics and Gynecology, \\ 800 Rose Street, C:360, Lexington, KY 40536 \\ (e-mail: niraj.chavan@uky.edu).
}

\begin{abstract}
Keywords

- buprenorphine

- opioid use disorder (OUD)

- medication assisted treatment (MAT)

- neonatal opioid withdrawal syndrome (NOWS)

Objective To examine the relationship between antepartum buprenorphine dose for medication-assisted treatment (MAT) of opioid use disorder (OUD) and incident neonatal opioid withdrawal syndrome (NOWS).

Study Design We performed a prospective cohort study of pregnant women with a singleton gestation diagnosed with OUD and receiving buprenorphine for MAT at a tertiary care academic institution from July 2015 to January 2017. We divided the study cohort into two groups-pregnancies with versus without NOWS. Substance abuse patterns in pregnancy, maternal, and neonatal clinical outcomes were compared.

Results The incidence of NOWS was $31.11 \%(n=28 / 90)$ in our study cohort. Pregnancies with NOWS had a significantly higher rate of benzodiazepine positive urine tests and number of positive urine drug screen (UDS) results for illicit opioids. The group without NOWS had significantly higher number of patients with an appropriate UDS result at delivery through postpartum. Rates of neonatal intensive care unit (NICU) admission, length of NICU stay, and maximum Finnegan score were significantly higher in the group with NOWS. Neither the initial $(10.6 \pm 5.2$ versus $10.3 \pm 4.8 \mathrm{mg}, p=0.80)$ nor the final buprenorphine doses $(13.3 \pm 5.1$ versus $13.0 \pm 4.6 \mathrm{mg}, p=0.81)$ were significantly different between study groups.

Conclusion The occurrence of NOWS was not related to buprenorphine dose used for MAT.
\end{abstract}

Opioid use disorder (OUD) is increasingly recognized as a national public health epidemic in the United States, leading not only to adverse perinatal outcomes, but also negative effects on childhood neurodevelopment. ${ }^{1-5}$ The problem is further compounded by the increasing burden of healthcare costs associated with treatment of neonates exposed to opioids in utero who then experience neonatal opioid withdrawal syndrome (NOWS). ${ }^{6,7}$ Management algorithms of OUD in pregnancy have traditionally hinged on the use of methadone as the agent of choice for pharmacotherapyeither constituted along the lines of a methadone "taper" or maintaining a steady state of methadone as a form of received

May 26, 2017

accepted after revision

October 9, 2017
DOI https://doi.org/

10.1055/s-0037-1608783. ISSN 2157-6998.
Copyright $\odot 2017$ by Thieme Medical Publishers, Inc., 333 Seventh Avenue, New York, NY 10001, USA. Tel: +1(212) 584-4662.
License terms

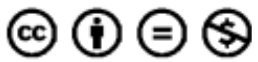


medication-assisted treatment (MAT) ${ }^{8-10}$ However, the use of methadone for MAT during pregnancy also carries the risk of fetal exposure in utero, manifesting as NOWS, also known as neonatal abstinence syndrome (NAS). ${ }^{10,11}$ Affected neonates exhibit a clinical profile characterized by hyperirritability, agitation, and central and/or autonomic nervous system dysfunction often necessitating intervention with pharmacologic therapy. This in turn leads to prolonged inpatient hospitalization for the neonate and the need for continued outpatient surveillance. ${ }^{10-15}$ Recently, attention has shifted toward the use of buprenorphine as an agent of choice for MAT of OUDs in pregnancy. Buprenorphine administration was initially targeted toward women who could be followed-up at longer intervals, representing a higher level of medication adherence and compliance, without the need for daily monitoring (as is the case with methadone). Buprenorphine treatment in an office-based setting allows women to have prescriptions for their medications, longer intervals between visits, and unsupervised dosing representing a model of care consistent with other chronic medical illnesses, in contrast to methadone treatment that requires more intensive monitoring with daily supervised dosing for the first 90 days of treatment that sometimes becomes a barrier to treatment entry. ${ }^{16-19}$ Importantly, maternal treatment with buprenorphine in pregnancy has been shown to have similar efficacy as methadone while also representing a safe and convenient option for outpatient management by allowing for prescribing through certified physician offices rather than daily attendance at methadone clinics. ${ }^{16,18,20,21}$ While buprenorphine is generally accepted in pregnancy as a safe alternative to methadone for MAT, the extent to which buprenorphine dose correlates with the risk of NOWS remains incompletely understood, with some studies supporting a dose-response relation and others not. 1,17,20-22

The objective of our study was to examine the relationship between antepartum buprenorphine dose among pregnant women undergoing MAT for OUD and incident NOWS.

\section{Methods}

The study was approved by the Institutional Review Board (IRB) at the University of Kentucky. Women were recruited from the Perinatal Assistance and Treatment Home (PATHways) Program at the University of Kentucky Polk Dalton clinic-an outpatient treatment program that includes group prenatal and postpartum care, MAT, and supportive services for high-risk women throughout Kentucky. We prospectively recruited and followed a cohort of pregnant women with a well-documented history of OUD, receiving prenatal care at a tertiary teaching institution from July 2015 to January 2017. Inclusion criteria included: (1) current diagnosis of opioid dependence with participation in the buprenorphine treatment program; (2) less than 30 weeks with a singleton gestation; and (3) age 18 to 49 years old. Women were excluded if they had current prisoner status, current severe mental illness (e.g., bipolar disorder with current mania, current suicidal ideation), or alcohol or sedative/hypnotic dependence that required medical intervention.
All patients received buprenorphine as the agent of choice for MAT of OUD, which was prescribed by waivered (X-licensed) maternal fetal medicine physicians with advanced training and licensure for buprenorphine administration. Buprenorphine dosing was once daily after initial buprenorphine induction, which verified objective withdrawal with the Clinical Opiate Withdrawal Scale (COWS). Medication adjustments were individualized for each patient based on healthcare provider clinical judgment, factoring in patient response and medication adherence. Patients received no more than a 2 -week prescription at any given point in time. All patients received buprenorphine in the form of the commercially marketed generic formulationSubutex (Reckitt Benckiser Pharmaceuticals, Inc, Richmond, Virginia), containing only buprenorphine, without naloxone. Pregnant patients with ongoing illicit drug use, e.g., as determined by self-report and/or urine drug testing, were managed by a customized approach centered upon escalation of care. For example, patients received increased face-toface counseling with providers having subspecialty training in maternal fetal medicine, mental health, and addiction medicine. This was accompanied by closer follow-up and surveillance monitoring with visits ranging from once to thrice weekly. Patients were followed through delivery until 6 weeks postpartum. Patients underwent serial urine drug screens (UDS) at each prenatal visit, upon admission into the Labor \& Delivery Unit and every postpartum visit. MAT was continued in all cases through the study period. Upon completion of the 6-week postpartum period, maternal and neonatal clinical outcomes data were abstracted through a review of their respective medical records. Data pertaining to maternal demographics, patterns of antenatal substance abuse, and incident pregnancy comorbidities were obtained from a thorough review of their outpatient electronic medical record. Labor and delivery-related outcomes were ascertained through a review of the inpatient electronic medical record system. The diagnosis of NOWS requiring pharmacologic management was confirmed based on documentation in the neonatal electronic medical record system. These cases were managed as per standardized institutional neonatal intensive care unit (NICU) protocols that used a modified Finnegan scale for scoring neonates for opioid withdrawal, also known as the MOTHER NAS scale. ${ }^{1,14}$ In this system, scoring is done for any infant chronically exposed to opioids in utero or who has demonstrated signs of significant withdrawal. Scoring is performed every 3 to 4 hours and changed to every 6 to 8 hours once the infant is stable. Pharmacologic therapy was initiated when three consecutive scores were $>8$ or two consecutive scores were $>12$. We divided the study cohort into two groups-that is, pregnancies with and without incident NOWS requiring pharmacotherapy.

Details of maternal substance abuse patterns during pregnancy, as well as maternal and neonatal clinical outcomes, were compared across these two groups, using the two-sample $t$-test or Mann-Whitney U test for continuous data, as appropriate, and the chi-square test of association or Fisher's exact test for categorical data. All statistical analyses were performed using SAS version 9.4; an $\alpha$ level of 0.05 was used throughout. 


\section{Results}

We recruited a total of 96 patients receiving buprenorphine for treatment of OUD in pregnancy. Of these, 90 patients delivered at our tertiary teaching hospital and were therefore available for follow-up of maternal and neonatal outcomes (the rest delivered at an outside hospital and hence their delivery outcomes data and neonatal data were unavailable and excluded from analyses). The incidence of NOWS was noted to be $31.11 \%$ in our cohort $(n=28)$. Demographic characteristics ( $\mathbf{- T a b l e ~ 1 )}$ ) were similar among the group of patients with and without incident NOWS. Interestingly, patterns of tobacco use during pregnancy (including smoking before and during pregnancy) as well as the use of smoking cessation treatment with nicotine replacement therapy were also similar in both groups ( $p>0.05)$.

Substance abuse characteristics between the two groups are compared in - Table 2 . Patients in each group underwent buprenorphine induction at $\sim 20$ weeks gestation. Routes of substance use or age at initiation of substance misuse did not differ between the two groups. The group with NOWS was noted to have a significantly higher rate of benzodiazepine abuse-as noted in their history of substance abuse as well as detection at initial UDS. Significantly, pregnancies with
NOWS were also noted to have a significantly higher number of positive UDS results (for illicit substances) over the course of pregnancy. Most importantly, neither the initial nor the final buprenorphine dose at delivery was significantly different between the two groups.

-Table 3 compares maternal pregnancy and delivery outcomes as well as postpartum course among the two study groups. There were no significant differences in the incidence of adverse perinatal outcomes between the study groups. However, the group without NOWS had a significantly higher number of patients with an appropriate UDS result (i.e., only positive for buprenorphine) at delivery, 2, 4, and 6 weeks postpartum. Rates of NICU admission, length of NICU stay, and maximum Finnegan score were significantly higher in the group with NOWS, as seen in - Table 4. The remainder of the neonatal outcomes under evaluation failed to reach statistical significance.

\section{Discussion}

Within our clinical cohort, the occurrence of NOWS was not related to the buprenorphine dose used for MAT of OUD in pregnancy. There were no significant differences in the initial or final buprenorphine dose in pregnancies with and without

Table 1 Demographic characteristics

\begin{tabular}{|c|c|c|c|c|}
\hline & $\begin{array}{l}\text { Total sample } \\
(N=90) \\
\text { Mean } \pm \text { SD, } \\
\text { Median (IQR) } \\
\text { or } n(\%)\end{array}$ & $\begin{array}{l}\text { NOWS } \\
(n=28) \\
\text { Mean } \pm \text { SD, } \\
\text { Median (IQR) } \\
\text { or } n(\%)\end{array}$ & $\begin{array}{l}\text { Non-NOWS } \\
(n=62) \\
\text { Mean } \pm \text { SD, } \\
\text { Median (IQR) } \\
\text { or } n(\%)\end{array}$ & $p$ \\
\hline Age & $28.8 \pm 4.6$ & $28.6 \pm 5.1$ & $28.9 \pm 4.4$ & 0.79 \\
\hline Gravidity & $3(2-5)$ & $3(2-4.5)$ & $3(2-5)$ & 0.98 \\
\hline Parity (living) & $2(1-3)$ & $2(1-2.5)$ & $2(1-3)$ & 0.86 \\
\hline Living children parent custody & $0(0-1)$ & $0(0-0.5)$ & $0(0-1.0)$ & 0.29 \\
\hline BMI at start of care & $25.1 \pm 4.6$ & $25.2 \pm 4.5$ & $25.0 \pm 4.6$ & 0.82 \\
\hline \multicolumn{5}{|l|}{ Race/ethnicity } \\
\hline White/non-Hispanic & $83(92.2 \%)$ & $25(89.3 \%)$ & $58(93.6 \%)$ & 0.67 \\
\hline Other & $7(7.8 \%)$ & $3(10.7 \%)$ & $4(6.4 \%)$ & \\
\hline \multicolumn{5}{|l|}{ Partnered status } \\
\hline Yes & $33(37.1 \%)$ & $10(37.0 \%)$ & $23(37.1 \%)$ & \multirow[t]{2}{*}{0.99} \\
\hline No & $56(62.9 \%)$ & $17(63.0 \%)$ & 39 (62.9\%) & \\
\hline \multicolumn{5}{|l|}{ Employment } \\
\hline Employed & $14(15.6 \%)$ & $1(3.6 \%)$ & $13(21.0 \%)$ & \multirow[t]{2}{*}{0.056} \\
\hline Unemployed & $76(84.4 \%)$ & 27 (96.4\%) & 49 (79.0\%) & \\
\hline \multicolumn{5}{|l|}{ Nicotine replacement } \\
\hline Yes & $20(24.4 \%)$ & $4(15.4 \%)$ & $16(28.6 \%)$ & \multirow[t]{2}{*}{0.20} \\
\hline No & $62(75.6 \%)$ & $22(84.6 \%)$ & 40 (71.4\%) & \\
\hline \multicolumn{5}{|l|}{ Using tobacco at delivery } \\
\hline Yes & $82(92.1 \%)$ & $26(92.9 \%)$ & $56(91.8 \%)$ & \multirow[t]{2}{*}{0.86} \\
\hline No & 7 (7.9\%) & $2(7.1 \%)$ & $8(8.2 \%)$ & \\
\hline
\end{tabular}

Abbreviations: BMI, body mass index; IQR, interquartile range; NOWS, neonatal opioid withdrawal syndrome; SD, standard deviation. Note: $p$-Values represent comparison between the NOWS and non-NOWS group. 
Table 2 Details of substance abuse patterns

\begin{tabular}{|c|c|c|c|c|}
\hline & $\begin{array}{l}\text { Total sample } \\
(N=90) \\
\text { Mean } \pm S D \text {, } \\
\text { Median (IQR) } \\
\text { or } n(\%)\end{array}$ & $\begin{array}{l}\text { NOWS } \\
(n=28) \\
\text { Mean } \pm \text { SD, } \\
\text { Median (IQR) } \\
\text { or } n(\%)\end{array}$ & $\begin{array}{l}\text { Non-NOWS } \\
(n=62) \\
\text { Mean } \pm \text { SD, } \\
\text { Median (IQR) } \\
\text { or } n(\%)\end{array}$ & $p$ \\
\hline Gestational age at program initiation & $21.5 \pm 8.4$ & $23.3 \pm 8.4$ & $20.6 \pm 8.4$ & 0.17 \\
\hline Gestational age at buprenorphine induction & $21.2 \pm 8.1$ & $22.1 \pm 7.6$ & $20.8 \pm 8.3$ & 0.53 \\
\hline Age at first substance abuse & $18.7 \pm 5.0$ & $18.5 \pm 5.9$ & $18.8 \pm 4.4$ & 0.80 \\
\hline \multicolumn{5}{|l|}{ History of substance abuse } \\
\hline Opioids & $88(97.8 \%)$ & $28(100.0 \%)$ & $60(96.8 \%)$ & $>0.99$ \\
\hline Methamphetamine & $17(18.9 \%)$ & $7(25.0 \%)$ & $10(16.1 \%)$ & 0.32 \\
\hline Cocaine & $25(27.8 \%)$ & $9(32.1 \%)$ & $16(25.8 \%)$ & 0.53 \\
\hline Benzodiazepines & $31(34.4 \%)$ & $13(46.4 \%)$ & $18(29.0 \%)$ & 0.11 \\
\hline THC & $54(60.0 \%)$ & $19(67.9 \%)$ & 35 (56.5\%) & 0.31 \\
\hline \multicolumn{5}{|l|}{ Route of substance abuse } \\
\hline Oral & $85(94.4 \%)$ & $26(92.9 \%)$ & $59(95.2 \%)$ & 0.65 \\
\hline Intravenous & $43(47.8 \%)$ & $16(57.1 \%)$ & $27(43.6 \%)$ & 0.23 \\
\hline Intranasal & $68(75.6 \%)$ & $26(92.9 \%)$ & $42(67.7 \%)$ & 0.015 \\
\hline \multicolumn{5}{|l|}{ Substances positive on initial UDS } \\
\hline Opioids & $34(37.8 \%)$ & $11(39.3 \%)$ & $23(37.1 \%)$ & 0.84 \\
\hline Methamphetamine & $7(7.8 \%)$ & $3(10.7 \%)$ & $4(6.5 \%)$ & 0.67 \\
\hline Cocaine & $10(11.1 \%)$ & $6(21.4 \%)$ & $4(6.5 \%)$ & 0.065 \\
\hline Benzodiazepines & $16(17.8 \%)$ & $10(35.7 \%)$ & $6(9.7 \%)$ & 0.006 \\
\hline THC & $27(30.0 \%)$ & $12(42.9 \%)$ & $15(24.2 \%)$ & 0.074 \\
\hline Positive UDS (\% yes) & $69(76.7 \%)$ & $27(96.4 \%)$ & $42(67.7 \%)$ & 0.003 \\
\hline $\begin{array}{l}\text { Number of positive UDS total } \\
\text { (any substance other than buprenorphine) }\end{array}$ & $4(1-8)$ & $9(6-12.5)$ & $2(0-6)$ & $<0.001$ \\
\hline Positive UDS illicit opioids (\% yes) & $48(53.3 \%)$ & $23(82.1 \%)$ & $25(40.3 \%)$ & $<0.001$ \\
\hline Number of positive UDS total - illicit opioids & $1(0-3)$ & $2(1-4)$ & $0(0-2)$ & $<0.001$ \\
\hline Initial buprenorphine dose & $10.4 \pm 4.9$ & $10.6 \pm 5.2$ & $10.3 \pm 4.8$ & 0.80 \\
\hline Final buprenorphine dose & $13.1 \pm 4.7$ & $13.3 \pm 5.1$ & $13.0 \pm 4.6$ & 0.81 \\
\hline
\end{tabular}

Abbreviations: IQR, interquartile range; NOWS, neonatal opioid withdrawal syndrome; SD, standard deviation; THC, tetrahydrocannabinol; UDS, urine drug screen.

Note: $p$-Values represent comparison between the NOWS and non-NOWS group.

incident NOWS. The incidence of NOWS in our cohort was noted to be $31.11 \%$, which is lower than the reported rates in several other contemporary studies. ${ }^{1,23,24}$ The incidence of NOWS requiring treatment in the setting of maternal buprenorphine use has been variably reported from 22 to $63 \% .^{21-26}$ Interestingly, several of these studies have focused on comparing the impact of methadone versus buprenorphine on incident NOWS. Patterns of tobacco use, including ongoing smoking and smoking cessation/nicotine replacement therapy, were similar among patients with and without incident NOWS in our study. This is in contrast to studies which show a relationship between maternal tobacco exposure and an increased likelihood of developing NOWS among women undergoing MAT for OUD in pregnancy. 27,28

In a double-blind randomized controlled trial called the Maternal Opioid Treatment: Human Experimental Research
(MOTHER) project, Jones et al compared neonatal outcomes among women undergoing MAT with methadone versus buprenorphine at eight different international sites. They found that neonates born to mothers receiving buprenorphine for MAT had a significantly shorter duration of treatment of NOWS and required significantly lesser morphine for treatment. ${ }^{1}$ However, the total number of neonates receiving treatment for NOWS was not statistically different in the two groups in this study. The incidence of treatment requiring NOWS in the group receiving buprenorphine in the MOTHER study was $47 \%$.

Globally, few other studies have delved into the evaluation of methadone versus buprenorphine and consequent development of NOWS. In a nonrandomized population-based Swedish study, Kakko et al compared neonatal outcomes among prospectively followed pregnancies exposed to buprenorphine with retrospectively analyzed pregnancies exposed to 
Table 3 Maternal pregnancy course, delivery, and postpartum outcomes

\begin{tabular}{|c|c|c|c|c|}
\hline & $\begin{array}{l}\text { Total sample } \\
(N=90) \\
n(\%)\end{array}$ & $\begin{array}{l}\text { NOWS } \\
(n=28) \\
n(\%)\end{array}$ & $\begin{array}{l}\text { Non-NOWS } \\
(n=62) \\
n(\%)\end{array}$ & $p$ \\
\hline Preterm labor & $11(12.2 \%)$ & $2(7.1 \%)$ & $9(14.5 \%)$ & 0.49 \\
\hline Preeclampsia & $5(5.7 \%)$ & $1(3.6 \%)$ & $4(6.7 \%)$ & $>0.99$ \\
\hline Gestational hypertension & $7(7.8 \%)$ & $1(3.6 \%)$ & $6(9.7 \%)$ & 0.43 \\
\hline Diabetes & $6(6.7 \%)$ & $4(14.3 \%)$ & $2(3.2 \%)$ & 0.073 \\
\hline IUGR & $10(11.1 \%)$ & $1(3.6 \%)$ & $9(14.5 \%)$ & 0.16 \\
\hline Oligohydramnios & $1(1.1 \%)$ & $0(0.0 \%)$ & $1(1.6 \%)$ & $>0.99$ \\
\hline Polyhydramnios & $1(1.1 \%)$ & $1(3.6 \%)$ & $0(0.0 \%)$ & 0.31 \\
\hline Hepatitis C & $42(47.2 \%)$ & $14(50.0 \%)$ & $28(45.9 \%)$ & 0.72 \\
\hline RPR positivity & $1(1.1 \%)$ & $1(3.6 \%)$ & $0(0.0 \%)$ & 0.31 \\
\hline Cholestasis & $2(2.9 \%)$ & $2(8.7 \%)$ & $0(0.0 \%)$ & 0.11 \\
\hline \multicolumn{5}{|l|}{ Mode of delivery } \\
\hline Primary cesarean section & $10(11.1 \%)$ & $3(10.7 \%)$ & $7(11.3 \%)$ & \multirow[t]{5}{*}{0.78} \\
\hline Repeat cesarean section & $29(32.2 \%)$ & $11(39.3 \%)$ & $18(29.0 \%)$ & \\
\hline Assisted vaginal & $2(2.2 \%)$ & $1(3.6 \%)$ & $1(1.6 \%)$ & \\
\hline Spontaneous vaginal & $48(53.3 \%)$ & $13(46.4 \%)$ & $35(56.5 \%)$ & \\
\hline VBAC & $1(1.1 \%)$ & $0(0.0 \%)$ & $1(1.6 \%)$ & \\
\hline \multicolumn{5}{|l|}{ Induction of labor } \\
\hline Yes & $24(27.3 \%)$ & $6(22.2 \%)$ & $18(29.5 \%)$ & \multirow[t]{2}{*}{0.48} \\
\hline No & 64 (72.7\%) & $21(77.8 \%)$ & $43(70.5 \%)$ & \\
\hline \multicolumn{5}{|l|}{ Augmentation of labor } \\
\hline Yes & $20(27.4 \%)$ & $6(26.1 \%)$ & $14(28.0 \%)$ & \multirow[t]{2}{*}{0.86} \\
\hline No & $53(72.6 \%)$ & $7(73.9 \%)$ & $36(72.0 \%)$ & \\
\hline \multicolumn{5}{|l|}{ Appropriate UDS at delivery } \\
\hline Yes & $59(67.8 \%)$ & $13(46.4 \%)$ & $46(78.0 \%)$ & \multirow[t]{3}{*}{0.002} \\
\hline No & $20(23.0 \%)$ & $13(46.4 \%)$ & $7(11.9 \%)$ & \\
\hline Not done & $8(9.2 \%)$ & $2(7.2 \%)$ & $6(10.2 \%)$ & \\
\hline UDS appropriate at $2 \mathrm{wk}$ postpartum $(n=82)$ & 60 (73.2\%) & $10 / 24(41.7 \%)$ & $50 / 58(86.2 \%)$ & $<0.001$ \\
\hline UDS appropriate at 4 wk postpartum $(n=76)$ & $54(71.1 \%)$ & $12 / 23(52.2 \%)$ & $42 / 53(79.3 \%)$ & 0.017 \\
\hline UDS appropriate at $6 \mathrm{wk}$ postpartum $(n=58)$ & $33(56.9 \%)$ & $8 / 21(38.1 \%)$ & $25 / 37(67.6 \%)$ & 0.029 \\
\hline
\end{tabular}

Abbreviations: IUGR, intrauterine growth restriction; NOWS, neonatal opioid withdrawal syndrome; RPR, rapid plasma reagin; UDS, urine drug screen; VBAC, vaginal birth after cesarean section.

Note: $p$-Values represent comparison between the NOWS and non-NOWS group.

methadone. In this study, treatment with buprenorphine offered the advantage of lower incidence of NOWS requiring pharmacological treatment, as well as shorter length of hospital stay for affected neonates. ${ }^{23}$ In a similar study of a Norwegian national clinical cohort of 139 mother-neonate couplets, Welle-Strand et al compared neonatal outcomes among patients receiving methadone versus buprenorphine for MAT during pregnancy. They found that the number of neonates that received treatment for NOWS was not significantly different between both groups as was the case with the peak Finnegan score and the length of treatment. ${ }^{24}$ Significantly, in a subgroup analysis, this group found that the concomitant benzodiazepines abuse during pregnancy while on MAT was associated with longer duration of treatment for NOWS. These findings parallel our observation wherein pregnancies affected by NOWS appear to have a significantly higher rate of benzodiazepine abuse. This also points toward the contributory role of concomitant illicit drug use (particularly benzodiazepine abuse) on possibly altering neonatal outcomes among pregnant women receiving MAT for OUD.

In a secondary analysis of the MOTHER study, Jones et al examined the relationship between buprenorphine at delivery among patients undergoing MAT and neonatal outcomes including NOWS. ${ }^{29}$ The authors found no relationship between buprenorphine dose and any of the neonatal outcomes under study, including incident NOWS, gestational age at delivery, 
Table 4 Neonatal course

\begin{tabular}{|c|c|c|c|c|}
\hline & $\begin{array}{l}\text { Total sample } \\
(N=90) \\
\text { Mean } \pm S D \text {, } \\
\text { Median (IQR) } \\
\text { or } n(\%)\end{array}$ & $\begin{array}{l}\text { NOWS } \\
(n=28) \\
\text { Mean } \pm \text { SD, } \\
\text { Median (IQR) } \\
\text { or } n(\%)\end{array}$ & $\begin{array}{l}\text { Non-NOWS } \\
(n=62) \\
\text { Mean } \pm \text { SD, } \\
\text { Median (IQR) } \\
\text { or } n(\%)\end{array}$ & $p$ \\
\hline Gestational age at delivery & $38.0 \pm 2.2$ & $38.5 \pm 1.1$ & $37.8 \pm 2.6$ & 0.17 \\
\hline \multicolumn{5}{|l|}{ Baby gender } \\
\hline Male & $43(48.9 \%)$ & 15 (53.6\%) & $32(53.3 \%)$ & \multirow[t]{2}{*}{0.55} \\
\hline Female & $45(51.1 \%)$ & $13(46.4 \%)$ & $28(46.7 \%)$ & \\
\hline \multicolumn{5}{|l|}{ APGAR score at $1 \mathrm{~min}$} \\
\hline$<7$ & $9(10.1 \%)$ & $4(14.8 \%)$ & $5(8.1 \%)$ & \multirow[t]{2}{*}{0.45} \\
\hline$\geq 7$ & $80(89.9 \%)$ & $23(85.2 \%)$ & $57(91.1 \%)$ & \\
\hline \multicolumn{5}{|l|}{ APGAR score at $5 \mathrm{~min}$} \\
\hline$<7$ & $6(6.7 \%)$ & $4(14.8 \%)$ & $2(3.2 \%)$ & \multirow[t]{2}{*}{0.066} \\
\hline$\geq 7$ & 83 (93.3\%) & $23(85.2 \%)$ & $60(96.8 \%)$ & \\
\hline Birth weight $(\mathrm{g})$ & $2804.3 \pm 491.8$ & $3013.6 \pm 350.4$ & $2709.7 \pm 519.0$ & 0.002 \\
\hline Birth weight percentile & $27.6 \pm 20.3$ & $38.5 \pm 23.2$ & $23.4 \pm 17.5$ & 0.003 \\
\hline \multicolumn{5}{|l|}{ Mode of feeding } \\
\hline Breastfeeding & 17 (18.9\%) & $2(7.1 \%)$ & 15 (24.2\%) & \multirow[t]{3}{*}{0.049} \\
\hline Bottle & $48(53.3 \%)$ & $20(71.4 \%)$ & $28(45.2 \%)$ & \\
\hline Combination & $25(27.8 \%)$ & $6(21.4 \%)$ & $19(30.7 \%)$ & \\
\hline NICU admission & $42(46.7 \%)$ & $28(100.0 \%)$ & $14(22.6 \%)$ & $<0.001$ \\
\hline Days in NICU & $6.5(0-17)$ & $16.5(12.5-23.5)$ & $0(0-5.5)$ & $<0.001$ \\
\hline Highest Finnegan score & $11(8-14)$ & $14(13-15)$ & $9(7-11)$ & $<0.001$ \\
\hline Maximum morphine dose & & $0.15(0.14-0.18)$ & & \\
\hline Total number of morphine doses & & $97(69-152)$ & & \\
\hline Infant UDS positive for buprenorphine & $68(80.0 \%)$ & $17(63.0 \%)$ & $51(87.9 \%)$ & 0.007 \\
\hline Infant UDS positive for norbuprenorphine & $75(88.2 \%)$ & $22(81.5 \%)$ & $53(91.4 \%)$ & 0.19 \\
\hline Maternal screen positive for buprenorphine & $51(58.6 \%)$ & $14(51.9 \%)$ & $37(67.7 \%)$ & 0.39 \\
\hline Maternal screen positive for norbuprenorphine & $74(85.1 \%)$ & $20(74.1 \%)$ & $54(90.0 \%)$ & 0.10 \\
\hline Breastfeeding at discharge & $31(34.8 \%)$ & $4(14.3 \%)$ & $27(44.3 \%)$ & 0.006 \\
\hline
\end{tabular}

Abbreviations: APGAR score, Appearance, Pulse, Grimace, Activity, and Respiration score; IQR, interquartile range; NICU, neonatal intensive care unit; NOWS, neonatal opioid withdrawal syndrome; SD, standard deviation; UDS, urine drug screen.

Note: $p$-Values represent comparison between the NOWS and non-NOWS group. All bold represents statistical significance at the $95 \%$ confidence level.

Appearance, Pulse, Grimace, Activity, and Respiration (APGAR) scores at 1 and 5 minute, neonatal head circumference, length and birth weight at birth, amount of morphine needed to treat NOWS, duration of treatment, and length of neonatal hospital stay. Based on these findings, this group failed to support the occurrence of a dose-response relationship between maternal buprenorphine dose for MAT and neonatal outcomes including NOWS. Paralleling these results, we failed to identify a relationship between buprenorphine dose and NOWS. In our study cohort, both the initial and final buprenorphine dose was similar among pregnancies with and without the occurrence of NOWS requiring pharmacologic treatment.

As a strength of our study, it prospectively followed patients receiving buprenorphine for MAT of OUD in pregnancy to evaluate the impact on maternal and neonatal outcomes, specifically NOWS. We also performed a detailed evaluation of patterns of substance abuse in our study cohort not only based on self-report but also ascertained through objective metrics such as UDS testing. Urine drug testing was performed throughout the course of pregnancy extending through the 6-week postpartum study period, and also included neonatal urine testing. Buprenorphine dosing was also managed by a single provider with specialized training and licensure with consultation provided as needed by an addiction medicine psychiatrist.

This study has limitations-first, we did not plan a priori to test the effect of varying dose levels of buprenorphine on NOWS; thus, we are unable to draw definitive conclusions about the buprenorphine dose-response relationship with incident NOWS. While the biologic plausibility of such a relationship 
seems appropriate, the current data are inadequate in addressing this question from an evidence-based standpoint. However, in our study, the buprenorphine dose was titrated to administer the most clinically appropriate dose on an individualized basis factoring in patient symptomatology and drug response, medication compliance, and UDS results. Hence, we were unable to specifically study the effect of varying dose levels. Similarly, group comparisons were not planned according to a specified level of statistical power, and post hoc power analysis is not valid. ${ }^{30}$ Future studies in this area may benefit from larger sample sizes for more powerful group comparisons. Second, alterations in buprenorphine dose were individualized, as against adopting a protocolized approach. Given the sheer paucity of data on alterations of buprenorphine metabolism over the pregnancy time course, we chose to adopt an individualized approach to identify the dose that best suited each patient's needs. Lastly, we are unable to comment on a comparison with methadone, since this was beyond the scope of this study. However, our data support the safety for adjusting the antepartum buprenorphine dose as deemed clinically appropriate with minimal neonatal adverse effects. Interestingly, there are inconsistent data regarding the relationship between antenatal buprenorphine dose in pregnancy for MAT and the severity of NOWS..$^{21,25,26}$ In an attempt to elucidate the biologic relationship between maternal buprenorphine and dose and incident NOWS, previous research has examined the levels of buprenorphine and its metabolites in the urine and meconium of infants born to mothers receiving buprenorphine during pregnancy. ${ }^{31,32}$ However, these studies provide limited insights into the plausibility of a relationship between antenatal buprenorphine dose and incident NOWS. In the light of these findings, our study aimed at examining the relationship between buprenorphine dose and incident NOWS among pregnant patients receiving buprenorphine for MAT of OUD during pregnancy. Based on our data, we are not able to establish a diagnosis of a relationship between buprenorphine dose in pregnancy and subsequent NOWS. However, our results suggest that women who are able to undergo MAT with buprenorphine in pregnancy without the concurrent use of illicit opioids or benzodiazepines are much less likely to have adverse neonatal outcomes as compared with their counterparts. This is important information for clinicians treating patients for OUD in pregnancy. Further studies-particularly, larger randomized prospective trials-are warranted to evaluate if a dose-response relationship exists between antenatal buprenorphine dose and the occurrence and severity of incident NOWS in pregnant patients undergoing MAT of OUD.

\section{Conflicts of Interest}

Dr. Lofwall reports grants and consulting fees from Braeburn Pharmaceuticals, consulting fees from Indivior, and honorarium from PCM Scientific, outside the submitted work.

\section{Financial Support}

This research did not have specific financial support.

Statement of Authorship

See attached documents.

\section{Note}

The abstract was presented at the 37th Annual Meeting of the Society of Maternal Fetal Medicine in January 2017Abstract \# 729.

\section{Condensation}

Examining the relationship between maternal buprenorphine dose for medication-assisted treatment of opioid use disorder of pregnancy and the occurrence of neonatal opioid withdrawal syndrome.

\section{References}

1 Jones HE, Kaltenbach K, Heil SH, et al. Neonatal abstinence syndrome after methadone or buprenorphine exposure. $\mathrm{N}$ Engl J Med 2010;363(24):2320-2331

2 Kandall SR, Albin S, Gartner LM, Lee K-S, Eidelman A, Lowinson J. The narcotic-dependent mother: fetal and neonatal consequences. Early Hum Dev 1977;1(02):159-169

3 Messinger DS, Bauer CR, Das A, et al. The maternal lifestyle study: cognitive, motor, and behavioral outcomes of cocaine-exposed and opiate-exposed infants through three years of age. Pediatrics 2004;113(06):1677-1685

4 Lifschitz MH, Wilson GS, Smith EO, Desmond MM. Factors affecting head growth and intellectual function in children of drug addicts. Pediatrics 1985;75(02):269-274

5 Women ACoHCfU. ACOG Committee Opinion No. 524: opioid abuse, dependence, and addiction in pregnancy. Obstet Anesthes Dig 2013;33:79-80

6 Center for Substance Abuse Treatment. Improving Treatment for Drug-Exposed Infants: Substance Abuse and Mental Health Services Administration(US); 1993. Report No.: (SMA) 93-2011. https://www. ncbi.nlm.nih.gov/pubmed/22514857. Accessed November 28, 2017

7 Patrick SW, Schumacher RE, Benneyworth BD, Krans EE, McAllister JM, Davis MM. Neonatal abstinence syndrome and associated health care expenditures: United States, 2000-2009. JAMA 2012; 307(18):1934-1940

8 Jones HE, O'Grady KE, Malfi D, Tuten M. Methadone maintenance vs. methadone taper during pregnancy: maternal and neonatal outcomes. Am J Addict 2008;17(05):372-386

9 Kaltenbach K, Berghella V, Finnegan L. Opioid dependence during pregnancy. Effects and management. Obstet Gynecol Clin North Am 1998;25(01):139-151

10 Dryden C, Young D, Hepburn M, Mactier H. Maternal methadone use in pregnancy: factors associated with the development of neonatal abstinence syndrome and implications for healthcare resources. BJOG 2009;116(05):665-671

11 Cleary BJ, Donnelly J, Strawbridge J, et al. Methadone dose and neonatal abstinence syndrome-systematic review and meta-analysis. Addiction 2010;105(12):2071-2084

12 Jansson LM, Velez M. Neonatal abstinence syndrome. Curr Opin Pediatr 2012;24(02):252-258

13 Bakstad B, Sarfi M, Welle-Strand GK, Ravndal E. Opioid maintenance treatment during pregnancy: occurrence and severity of neonatal abstinence syndrome. A national prospective study. Eur Addict Res 2009;15(03):128-134

14 Finnegan LP, Connaughton JF Jr, Kron RE, Emich JP. Neonatal abstinence syndrome: assessment and management. Addict Dis 1975;2(1-2):141-158

15 Jansson LM, Velez M, Harrow C. The opioid-exposed newborn: assessment and pharmacologic management. J Opioid Manag 2009;5(01):47-55

16 Fudala PJ, Bridge TP, Herbert S, et al; Buprenorphine/Naloxone Collaborative Study Group. Office-based treatment of opiate addiction with a sublingual-tablet formulation of buprenorphine and naloxone. N Engl J Med 2003;349(10):949-958 
17 Johnson RE, Jones HE, Fischer G. Use of buprenorphine in pregnancy: patient management and effects on the neonate. Drug Alcohol Depend 2003;70(2, Suppl)S87-S101

18 Jones ES, Moore BA, Sindelar JL, O'Connor PG, Schottenfeld RS, Fiellin DA. Cost analysis of clinic and office-based treatment of opioid dependence: results with methadone and buprenorphine in clinically stable patients. Drug Alcohol Depend 2009; 99(1-3):132-140

19 Jones HE. Practical considerations for the clinical use of buprenorphine. Sci Pract Perspect 2004;2(02):4-20

20 Jones HE, Finnegan LP, Kaltenbach K. Methadone and buprenorphine for the management of opioid dependence in pregnancy. Drugs 2012;72(06):747-757

21 Jones HE, Johnson RE, Jasinski DR, et al. Buprenorphine versus methadone in the treatment of pregnant opioid-dependent patients: effects on the neonatal abstinence syndrome. Drug Alcohol Depend 2005;79(01):1-10

22 Jones HE, Fischer G, Heil SH, et al. Maternal Opioid Treatment: Human Experimental Research (MOTHER)-approach, issues and lessons learned. Addiction 2012;107(Suppl 1):28-35

23 Kakko J, Heilig M, Sarman I. Buprenorphine and methadone treatment of opiate dependence during pregnancy: comparison of fetal growth and neonatal outcomes in two consecutive case series. Drug Alcohol Depend 2008;96(1-2):69-78

24 Welle-Strand GK, Skurtveit S, Jones HE, et al. Neonatal outcomes following in utero exposure to methadone or buprenorphine: a National Cohort Study of opioid-agonist treatment of Pregnant Women in Norway from 1996 to 2009. Drug Alcohol Depend 2013;127(1-3):200-206
25 Fischer G, Ortner R, Rohrmeister K, et al. Methadone versus buprenorphine in pregnant addicts: a double-blind, doubledummy comparison study. Addiction 2006;101(02):275-281

26 Lejeune C, Simmat-Durand L, Gourarier L, Aubisson S; Groupe d'Etudes Grossesse et Addictions (GEGA). Prospective multicenter observational study of 260 infants born to 259 opiate-dependent mothers on methadone or high-dose buprenophine substitution. Drug Alcohol Depend 2006;82(03):250-257

27 Choo RE, Huestis MA, Schroeder JR, Shin AS, Jones HE. Neonatal abstinence syndrome in methadone-exposed infants is altered by level of prenatal tobacco exposure. Drug Alcohol Depend 2004;75 (03):253-260

28 Winklbaur B, Baewert A, Jagsch R, et al. Association between prenatal tobacco exposure and outcome of neonates born to opioid-maintained mothers. Implications for treatment. Eur Addict Res 2009;15(03):150-156

29 Jones HE, Dengler E, Garrison A, et al. Neonatal outcomes and their relationship to maternal buprenorphine dose during pregnancy. Drug Alcohol Depend 2014;134:414-417

30 Hoenig JM, Heisey DM. The abuse of power: the pervasive fallacy of power calculations for data analysis. Am Stat 2001;55:19-24

31 Kacinko SL, Jones HE, Johnson RE, Choo RE, Huestis MA. Correlations of maternal buprenorphine dose, buprenorphine, and metabolite concentrations in meconium with neonatal outcomes. Clin Pharmacol Ther 2008;84(05):604-612

32 Hytinantti T, Kahila H, Renlund M, Järvenpää AL, Halmesmäki E, Kivitie-Kallio S. Neonatal outcome of 58 infants exposed to maternal buprenorphine in utero. Acta Paediatr 2008;97(08): 1040-1044 